\title{
Voices of biotech research
}

\author{
Nature Biotechnology asks a selection of faculty about the most exciting frontier in their field and the most needed \\ technologies for advancing knowledge and applications.
}

W

hat will be the most important areas of research in biotech over the coming years? Which technologies will be most important to advance knowledge and applications in these areas? Nature Biotechnology reached out to a set of faculty doing outstanding work in research areas representative of the journal's remit and asked them to contribute their vision of where their fields are going.

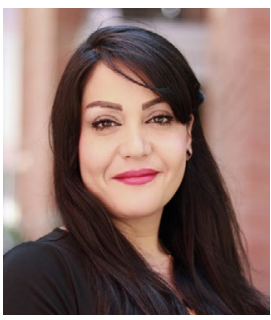

Nasim Annabi:

Bioengineering

advances have

increased

foundational

interdisciplinary

research for creating

state-of-the-art

medical devices and drug delivery

platforms; however, there are many challenges in the design and development of reliable technologies. The future trends in this field should focus on dynamic biomaterials, personalized medicine and additive manufacturing to improve both disease diagnostics and treatments. In addition, there is a need for the development of more effective in vitro platforms for developing drugs and testing medical devices to transform the healthcare system through preclinical research and its rapid scale-up and commercialization for effective and safe healthcare solutions.

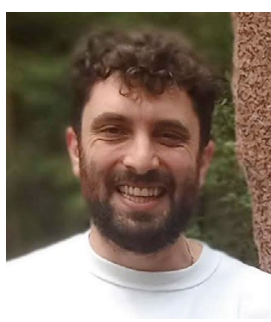

Matthew Baker: I have an interest in directed evolution and evolutionary microbiology. The 2018 Nobel Prize, partially awarded to Frances Arnold, has bolstered widespread interest

in utilizing evolution for approaches in synthetic biology. Major improvements have been made using evolutionary methods for 'irrational design' to test and engineer new proteins for new purposes. However, our understanding of emergent complexity - or how complexity increased greatly at certain key moments - remains limited. Experimental evolution is being applied to more complex systems and different species with better screens and higher throughput via automation. This should lead to a better understanding of what the broad 'rules' of molecular evolution are and, in turn, refine efforts in synthetic biology and directed evolution of proteins for applications in biotechnology.

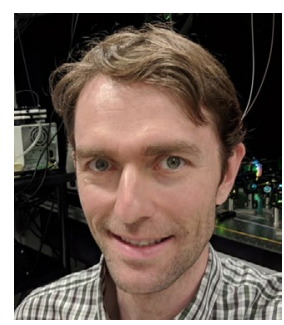

Alistair Boettiger:

Advances in sequence-resolved super-resolution imaging of the genome are changing how we think about chromatin structure and its roles in cell biology. It is now apparent that the genome doesn't fold into a stable structure, the way many proteins do. It is a large and flexible molecule, in which no two cells adopt the same fold at any given point in time. Understanding how this flexibility facilitates genome processes like transcriptional regulation, replication and repair will be driven forward by deeper integration of microscopy and sequencing approaches, new multimodal 'omic imaging, and microscopy-informed computational modeling.

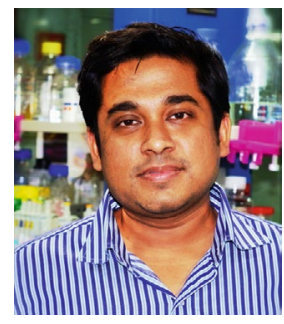

\section{Debojyoti} Chakraborty: Detecting and correcting diseases requires precise molecular tools. The promise shown by ongoing gene editing trials for hemoglobinopathies

has truly put CRISPR on track for therapeutic interventions. With the development of novel editors, cleavage-free genome engineering and robust delivery options, the coming years would see active clinical evaluation of in vivo genome editing - a challenging frontier. At the same time, the evolution of more sensitive and inexpensive CRISPR diagnostics platforms suited to a wide range of diseases would bring the benefits of early detection of disorders. This may be invaluable in developing countries.

Yvonne Chen: Cell-based immunotherapy has broken new grounds in treating

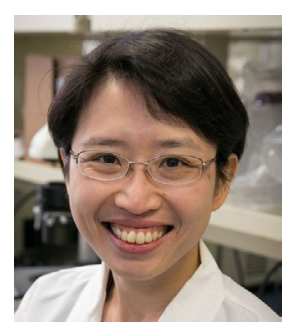

previously intractable diseases. Efforts in biomolecular engineering and synthetic biology continue to enhance our ability to engineer synthetic proteins that predictably impact cell behavior, moving the field away from a trial-and-error basis and toward true rational design. The development and integration of multilayered genetic circuitry offer the possibility of greater versatility and control over engineered cells. To reach their maximum potential, such designs must also be grounded in the realities of clinical implementation and engineered with an eye toward system robustness in the face of significant variabilities in human physiology.

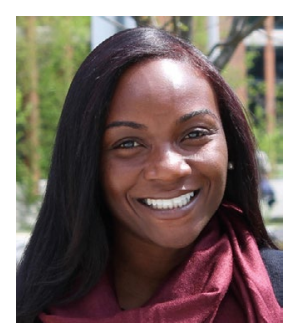

Kizzmekia S.

Corbett: As the COVID-19 pandemic has beckoned for rapid development of safe and effective vaccines, vaccinology is being transformed before our eyes. Now, the looming question for the field is not "Will novel viruses arise?" but "How can we be better prepared when they do?" The use of mRNA platforms awakens a new era of vaccine development that will rely on a critical intersection of basic science, precise antigen design, novel platform discovery and concerted global efforts towards pandemic preparedness.

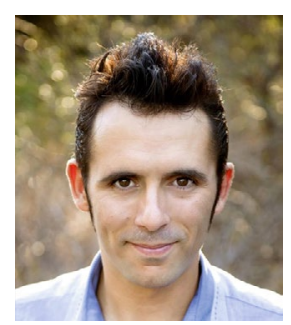

Bruno Correia: We continue to witness incredible advances in the broad field of molecular design. The ever-larger (and growing) data reservoirs, empowered both by unprecedented advances in computational analysis and by our ability to extract functional principles, are having a transformative impact. In the area of protein design, I foresee an 
increasing capacity to engineer extra mechanistic layers into amino acid sequences, allowing us to create 'smarter drugs' with multiple controllable activities. Another exciting dimension is where protein design interfaces with cell-based therapies, which will open up many new important avenues for next-generation therapies.

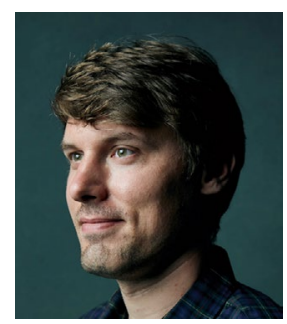

James Dahlman: Millions of patients have been treated via lipid nanoparticle (LNP)-mediated mRNA delivery; however, this emerging drug modality is limited to intramuscular administration or systemic liver targeting. To realize the clinical potential of RNA drugs, we must design LNPs that target new tissues after systemic administration, which likely means analyzing thousands of chemically distinct LNPs directly in vivo. DNA-barcoded nanoparticles make this increasingly plausible, enabling study of more than 100 LNPs in a single animal. The key lesson from DNA-barcoded nanoparticles, which should be applied to drug delivery in the future, is that bioengineering technologies used to perturb and characterize cells (for example, single-cell RNA-seq and multi-omics) can be repurposed to study targeted, systemic delivery in exciting new ways.

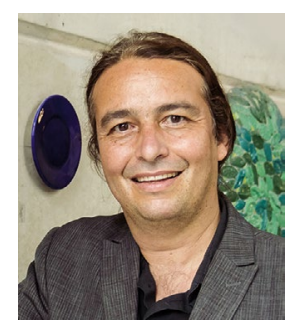

Tulio de Oliveira: In the field of genomic surveillance, one of the most exciting developments is genotype-tophenotype characterization in real time. For example, within weeks of the discovery of novel SARS-CoV-2 variants, researchers were able to determine how the genetic variation affected vaccine response.

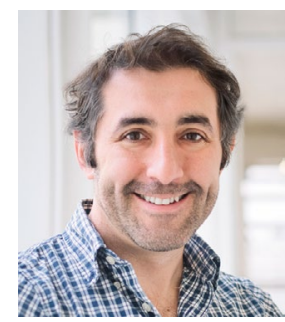

Ali Ertuerk: Moving from generalized and flawed treatments to personalized medicine requires merging unbiased and scalable technologies. Advances in single-cell

multi-omics and organoid models have facilitated the assessment of patient heterogeneity. By combining these technologies with unbiased imaging of intact biological specimens using tissue clearing and end-to-end artificial intelligence (AI)-based analysis, we can achieve a much-needed million-fold scaling of human organ mapping and investigation of diseases at the single-cell level. Although DNA nanotechnology can help target desired cells in vivo for drug delivery or gene editing, the cellular maps can serve as templates to generate new human organs using three-dimensional (3D) bioprinting for millions in need. This multidisciplinary approach will fast-track personalized treatments of complicated diseases and enable healthy living beyond 100 years.

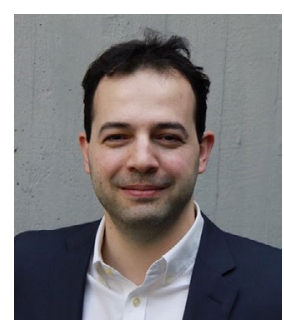

Mehmet Fatih Yanik: In the near future, implantable brain-machine interfaces based on biocompatible materials will be substantially less invasive while covering more brain areas and allowing neuronal-resolution measurements of rapidly varying brain networks. Technologies like focused ultrasound will be miniaturized and make it possible to focally deliver receptor-specific drugs non-invasively to specific circuits deep within the brain without causing off-target effects. Closed-loop implementation of such measurement and manipulation capabilities using AI algorithms to simultaneously analyze both brain networks and behavior are likely to enable unique opportunities for the treatment of brain disorders. These advances will present interesting regulatory, legal and ethical challenges.

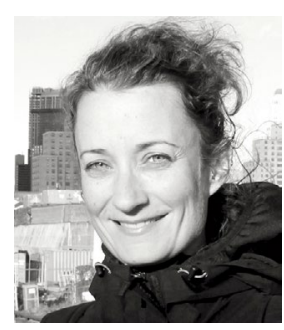

Elizabeth Henaff: The individual microbiome manifests the continuum between organism and environment. The next frontier of biotechnology is putting biological metrics, such as metagenomics, in the hands of the designers who create our built environments: architects and city planners. How can the discipline of design, an inherently human-centered practice, learn from the field of metagenomics? These metrics help us contextualize human health and well-being within the multispecies ecosystems we inhabit. Design for humans will become design for the more-than-human. The key will be to focus on relational and radical inclusion, with biotechnological interfaces designed for collaborative survival across scales and species.

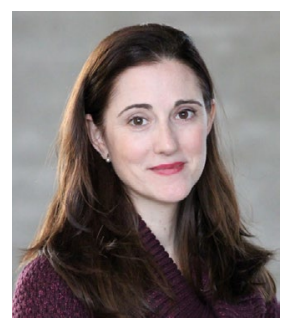

Meritxell Huch: One of the most exciting developments in human cell biology has been the establishment of 'organoids' - human 3D cultures that faithfully recapitulate some of the function

of the corresponding tissue. Derived either from pluripotent stem cells, by a stepwise differentiation process, or from differentiated (either adult or fetal) tissue, these self-organizing structures provide a unique system wherein to identify basic principles of human organ development, tissue regeneration and disease. Their ability to recapitulate organogenesis is revolutionizing the way we study human development. Similarly, the remarkable expansion potential of human organoid cultures derived from adult or fetal cells is opening up opportunities for biomedical research applications, ranging from cellular sources for cell therapy or transplantation to drug screening platforms for personalized medicine. Many challenges remain, ranging from increasing cellular complexity to improving scalability or cellular maturation. Once surmounted, we will enter a new era where studying basic principles of organ development, maintenance, regeneration and disease in human tissues will become a reality.

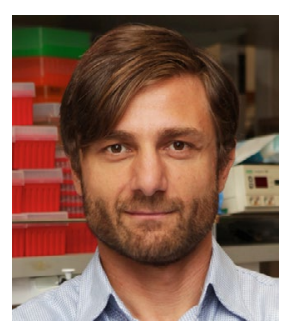

Iliyan D. Iliev: For many years, fungal research has been an important driving force in biotechnology and medicine. Recent discoveries place fungi as an integral part of the microbiome, key modulators of immunity and health, and rich sources of biologically active metabolites. Aided by advances in 'omics technologies, data integration and experimental platforms and a revolution in genome editing, we are now poised to decipher fungal activities and host-fungal interactions at an unprecedented level in individual patients. New therapeutic developments modulating such interactions or targeting the fungal arm of the microbiome will be the next challenge. 


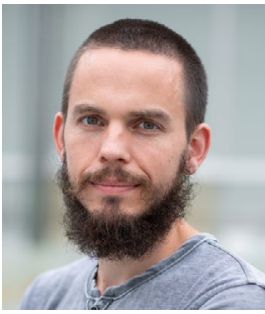

Credit: (C) VIB-Ine

Dehandschutter
Thomas Jacobs: One of the most exciting developments I see is the ability to make precise, large-scale perturbations to eukaryotic genomes. From combinatorial genetic screens to synthesis and reorganization on a large scale, we are getting closer to having the ability to precisely modify entire genomes of organisms at will. This will allow us to test hypotheses on genome structure, organization and gene regulatory networks in the wet lab, with applications in agriculture and biomedicine. To accomplish this, we will need to develop both methods for more accurate DNA synthesis that are orders of magnitude cheaper, and simple bioinformatic tools so the average student or postdoc can easily design, analyze and interpret these increasingly combinatorial experimental systems.

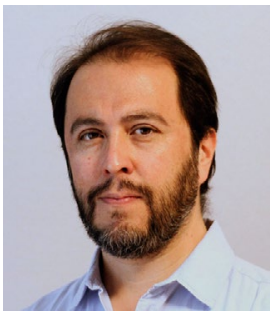

Howard Junca: Biomarkers, bioactivities and bioprocesses can contribute to achieving sustainability in the Anthropocene: a thrilling and challenging

time for environmental biotechnology and microbiome research. 'One Health' approaches to critical trends - rewilding Earth's ecosystems and functional restoration, xenobiotic containment and bioremediation, changes in production and consumption of goods, and responses to emerging diseases - all can greatly benefit by integrating microbiome engineering and transplantation. Disruptive computational power and approaches for biodata mining and AI pattern recognition will boost detection, cultivability, prediction and design of novel natural and synthetic components, metabolic network interdependencies and their combinatorial effects inside microbiomes and in holobiont associations.

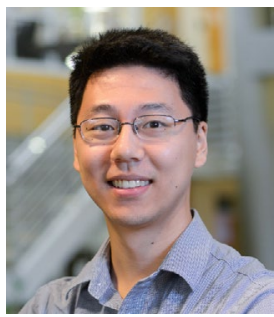

\section{Albert Keung:} Synthetic biology has accomplished many wonderful things by integrating our rapidly expanding understanding of biological parts and their functions into sophisticated systems. One intriguing question is how we might address problems that require substantial scaling. Although traditional engineering disciplines have developed frameworks to guide scaling, biological systems - often within what one would find in just a microliter of fluid - can present orders of magnitude higher levels of distinct and diverse intermolecular interactions than the largest chemical process industrial plant. Engineering regulatory systems within cells or storing exabytes of digital information in a billion, billion distinct DNA strands are just a few examples of extreme scaling that will demand new design and engineering frameworks.

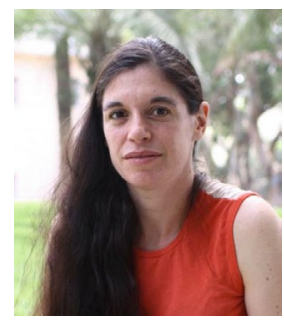

Ilana Kolodkin-Gal: In ancient Greece, Socrates and Plato founded Western scientific philosophy by integrating logic, poetry, math and natural sciences. The current renaissance of microbial

biotechnology similarly erases the artificial boundaries between genetics, chemistry, data science, ecology and even social sciences to utilize natural bacterial abilities. Microbial enzymes and exopolymers are used in biomedical and food industries while bacterial communal properties are explored to generate living concrete, novel drugs and higher crop productivity. Modern microbial biotechnology can overcome reactionary responses to the vital synthesis of traditional disciplines. Furthermore, it demonstrates that the coevolution of academy and industry is not a compromise, but rather an essential step for advancing scientific excellence.

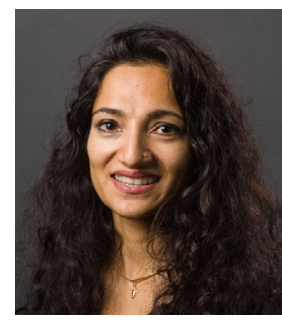

Smita

Krishnaswamy: Biomedical data are being generated at an extremely high throughput and in many dimensions. Initial phases of analysis involved tasks such as denoising, batch correction and basic dimensionality reduction. However, there is a big gap between data analysis and insight generation. I believe that the next phase will involve integrated analysis of multitudes of related datasets collected under many conditions and across modalities instead of an isolated focus on individual datasets. This will allow us to model the underlying systems as stochastic, complex and dynamic entities. From such models, we could infer mechanistics and even simulate potential therapies or alterations of the system.

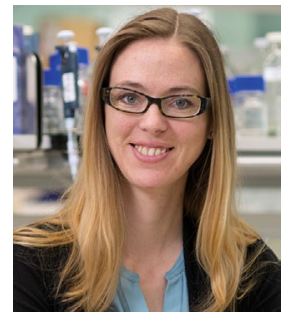

Madeline

Lancaster: Exciting discoveries in human neurobiology will come from the intersection of highly diverse methods, from genetics and transcriptomics to imaging and electrophysiology. These approaches will enable detailed characterization of cell types and neuronal connectivity. In this context, brain organoids will provide a tractable system for further information across time and even across species. But such neural tissues are of particular interest for their use in functional studies, allowing one to test resultant hypotheses through genetic or other perturbations. These varied approaches will begin to provide a mechanistic understanding of human brain development and function.

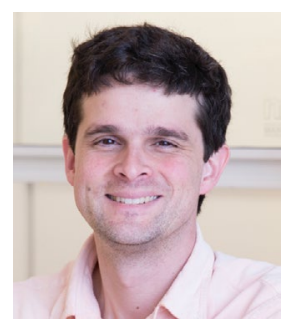

Evan Macosko: The use of DNA to barcode and quantify biomolecules powered the recent 'single cell revolution'. In combination with new developments in microscopy and molecular biology,

DNA will increasingly be the readout of choice for biochemical and cell biological assays in situ. Protein-protein interactions, cell signaling dynamics and metabolic state will all be encodable in DNA, enabling multiplexed, high-throughput measurements. In the brain, application of these technologies to the study of neural connectivity, electrical activity and functional plasticity will be transformative. I hope such assays finally provide the level of detail needed to understand the many brain diseases whose mechanisms remain mysterious.

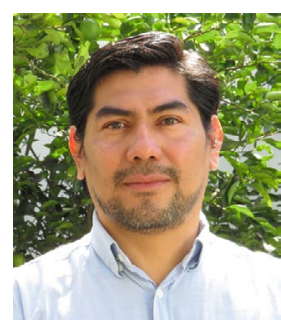

Mario Alberto Martínez-Núñez: The development of omics technologies and their coupling to synthetic biology will allow in the near future not only the exploration or modeling

of microbiomes for the search for environmental or biotechnological solutions, 
but also their control. The union and development of these fields will allow us to go from ex situ experiments to in situ implementations, such as the design, construction and control of communication between the elements of the microbiomes, allowing us to regulate not only cell behavior, but also production of molecules of interest in situ.

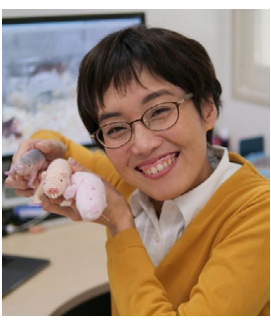

\section{Kyoko Miura:}

Research on long-lived species, such as naked mole-rats, blind mole-rats, bats, whales, elephants and long-lived trees, presents unique opportunities for identifying novel strategies to prevent aging and age-related diseases, including cancer. We believe that the development of genome editing, induced pluripotent stem (iPS) cells, and multi-omics technologies in the naked mole-rat, the longest-lived rodent, could pave the way to a fundamental cure for aging and cancer in humans. Combining studies in this new animal model with the rapidly progressing human iPS cell and organoid technologies could ultimately allow treatment of a variety of diseases and injuries.

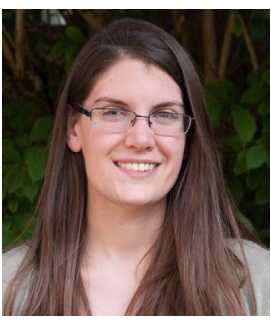

\section{Jenny Molloy:} During COVID19 , the impact of biotechnology on our daily lives and incredible recent advances in bioengineering, biomanufacturing and bioinformatics

have never been more visible. However, not everyone can access biotechnology's benefits - partly because the tools and agency to shape the field are very unevenly distributed. This has to change. Nurturing talent and ideas from all parts of the world is essential for a thriving and equitable global bioeconomy. We have the means: enabling technologies are being democratized, digital tools for sharing know-how are flourishing and open sharing of data and materials is accelerating. We will see a more diverse biotech community emerging that benefits even more of the planet.

Andrés Ochoa Cruz: Discovery has always been part of the human spirit. Coronavirus has opened the discussion of and interest in understanding science all over the globe. Involving people in scientific understanding is one of the pillars of the citizen-science

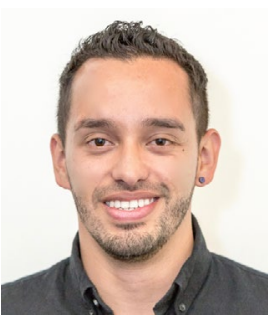

movement. I am excited about technologies and tools that are helping us to understand biology more deeply: synthetic biology that merges engineering, biology and mathematics;

machine learning that helps us to make sense of the large amount of data that we produce in genomics; and finally, self-quantification and health data analysis that can encourage greater public engagement with science and help people understand their own biology.

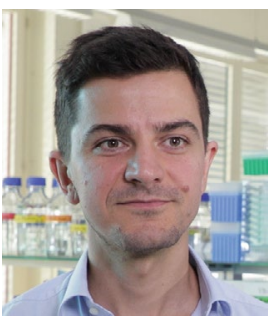

Randall J.

Platt: Fueled by

advancements

in biological engineering, DNA sequencing and DNA data storage, it is now possible to encode biological events in DNA and

subsequently reconstruct cellular histories providing an entry point into understanding the relationship between cellular lineages, transcriptomes, interactions and environments. The development of new molecular tools enabling the scalable encoding and integration of multimodal features within single cells and across multicellular systems will usher biology into a radically new era where biological systems can tell you their complex biographies spanning time, space, development, perturbation, health and disease.

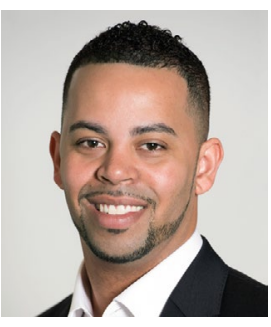

Avery D. Posey, Jr.: Cellular immunotherapy demonstrated ground-breaking advances for the treatment of blood cancers and budding activity against solid malignancies over the past decade. In the next decade, important objectives for improving the efficacy of cell therapy will be to identify optimal cell types and sources, expand tumor targeting to exploit differential post-translational modifications, revisit immune signaling pathways to identify opportunities to enhance potency and resist exhaustion, and alleviate immunosuppressive microenvironments through combination therapies, including novel synthetic molecules. Multi-omics technologies will improve our understanding of the factors driving responses to cell therapies and the relationship between innate, adaptive and engineered immunity within tumors.

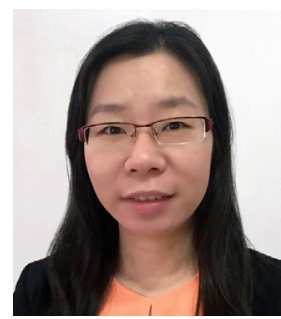

Huilin Shao:

I am most excited about the development of new nanotechnologies biological, physical and integrative - to empower molecular diagnostics. Improved precision in their design and engineering will bring forth enabling tools for basic discovery and clinical translation. These can redefine biomarkers, revealing insights from the currently unmeasurable, and influence patient care, through earlier and safer diagnostics and real-time treatment monitoring.

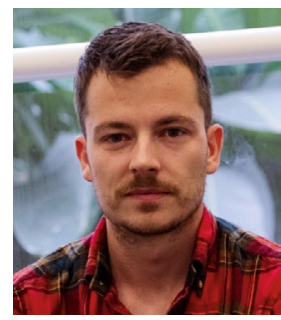

Mijo Simunovic: Organoids have created unique opportunities for modeling mammalian development in the lab, leaping into a new era of synthetic organogenesis.

Although pathways to generating individual organs are strikingly similar across many species, the human embryo is a world apart from a mouse or an elephant. Our next challenge is to leverage tissue engineering and genome editing to faithfully mimic the complex signaling gradients and the mechanical microenvironments of developing human organs. These efforts will not only enable a rationally designed program to reproducibly generate human tissues from pluripotent stem cells, but also open doors to answering perhaps one of the oldest questions in biology: what makes us human?

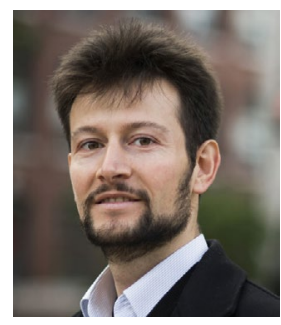

Nikolai Slavov: Single-cell multi-omics technologies are rapidly expanding. An exciting frontier is the comprehensive quantification of protein activities, interactions and conformations with single-cell resolution across time and space. Such analyses will be powered by emerging single-cell mass spectrometric technologies. These 
technologies will build quantitative biochemical and biophysical models of cellular systems and discover new drug targets. The success of such analyses will require (i) careful selection and sampling of relevant in vivo systems, including patient samples, and (ii) integration of multi-omics methods that analyze both cells and their environments. Areas poised to benefit from these developments include immuno-oncology, autoimmunity, neuroscience and developmental biology.

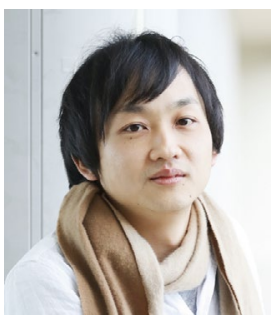

Takanori Takebe: Personalization 'My Medicine' will shape the future of medical practice and push humanity towards a better life. The advent of organoid research has offered a platform to study human health and disease, but going forward the technological convergence of evolving toolboxes, such as gene editing, transcriptomics, imaging and bioengineering, will act synergistically to enable personalized prediction of disease onset, prevention of disease, improved efficacy and safety of medical interventions and, ultimately, personalized regenerative therapy as human organoid research progresses.

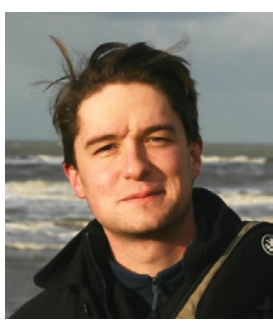

Luk H. Vandenberghe: The era of genetic medicine has started. The past years have shown that gene therapy can have a durable and transformative impact on disease and patients' lives. What is in front of us is exciting on many levels. For one, with the innovation in omics, the knowledge base of the targets we can pursue increases daily. Second, the creativity explosion on how we can molecularly intervene at those disease targets by editing, silencing or augmenting approaches is bringing a precision to the design table that often is critical to make a meaningful gene drug. Third, gene delivery, the focus of much of our work, often remains the Achilles heel to success in the clinic. Here too we see novel modalities, such as non-viral gene delivery (even mRNA for vaccines) and refinement of existing ones such as adeno-associated virus (AAV) to improve pharmacological control of this new class of drugs.

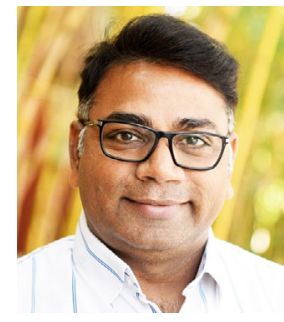

Rajeev K. Varshney:

The advent of contemporary genome sequencing tools and platforms have enabled researchers to sequence most of the crops and plant species,

including so-called orphan crops in developing countries. This has led to a better understanding of the genome architecture and the molecular basis of traits of interest for developing improved crop varieties addressing climate change, food, health, and nutrition security. We anticipate generation of massive amounts of sequence data not only at the single-cell level in plants, but also at the population level from the field. It will be exciting to see how of artificial intelligence and machine learning are applied to these datasets to enhance accuracy of genomic prediction for accelerated crop improvement programs.

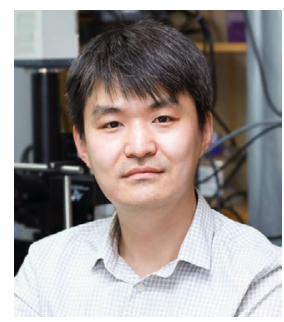

Jianbin Wang: Single-cell transcriptomics has propelled the identification of cell types in various organs and systems. Now basic and clinical fields are looking beyond cell type and expecting a more comprehensive understanding of cellular status in primary tissue. This requires not only gene expression profiles, but more importantly cell signaling cascades from receptor activation to transcription factor binding, which should be integrated with histology and pathology information at single-cell resolution. Technology breakthroughs for precise measurement of various biomolecules are necessary to achieve this goal.

\begin{tabular}{|c|}
\hline 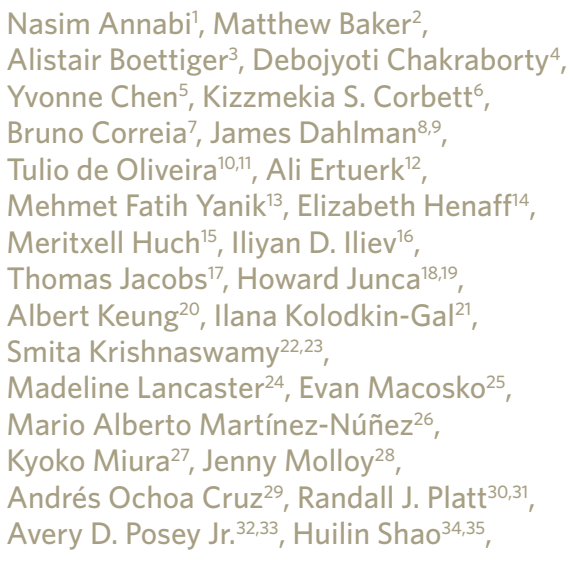 \\
\hline
\end{tabular}

Mijo Simunovic ${ }^{36,37}$, Nikolai Slavov 38,39,40, Takanori Takebe ${ }^{41}$, LukH. Vandenberghe ${ }^{25,42,43}$, Rajeev K. Varshney ${ }^{44,45}$ and Jianbin Wang 46

${ }^{1}$ Chemical and Biomolecular Engineering, UCLA Samueli School of Engineering,

Los Angeles, CA, USA. ${ }^{2}$ School of Biotechnology and Biomolecular Sciences at the University of New South Wales, Sydney, New South Wales, Australia. ${ }^{3}$ Department of Developmental Biology, Stanford University, Stanford, CA, USA. ${ }^{4}$ CSIR-Institute of Genomics and Integrative Biology, New Delhi, India. ${ }^{5}$ Department of Microbiology, Immunology and Molecular Genetics, University of California Los Angeles, Los Angeles, CA, USA. ${ }^{6}$ Viral Pathogenesis Laboratory, Vaccine Research Center, National Institutes of Allergy and Infectious Diseases, Bethesda, MD, USA. ${ }^{7}$ The Laboratory of Protein Design and Immunoengineering, Ecole Polytechnique Fédérale de Lausanne, Swiss Institute of Bioinformatics, Lausanne, Switzerland. ${ }^{8}$ Georgia Institute of Technology, Atlanta, GA, USA. ${ }^{9}$ Emory University School of Medicine, Atlanta, GA, USA. ${ }^{10} \mathrm{KwaZulu-Natal} \mathrm{Research} \mathrm{Innovation}$ and Sequencing Platform (KRISP), University of KwaZulu-Natal, Durban, South Africa. ${ }^{11}$ Department of Global Health, University of Washington, Seattle, WA, USA. ${ }^{12}$ Institute of Tissue Engineering and Regenerative Medicine, Munich Center for Neuroscience, Neuherberg, Germany. ${ }^{13}$ Department of Information Technology and Electrical Engineering, ETH, Zürich, Switzerland. ${ }^{14}$ Integrated Design and Media, Center for Urban Science and Progress, NYU Tandon School of Engineering, Brooklyn, NY, USA.

${ }^{15}$ Max Planck Institute of Molecular Cell Biology and Genetics, Dresden, Germany. ${ }^{16}$ Weill Medical College of Cornell University, New York, NY, USA. ${ }^{17}$ VIB University of Ghent Center for Plant Systems Biology, Ghent, Belgium. ${ }^{18}$ Microbiomas Foundation, Chia, Colombia. ${ }^{19} \mathrm{Helmholtz}$ Centre for Infection Research, Braunschweig, Germany. ${ }^{20}$ Chemical and Biomolecular Engineering, North Carolina State University, Raleigh, NC, USA. ${ }^{21}$ Department of Molecular Genetics, Weizmann Institute of Science, Rehovot, Israel. ${ }^{22}$ Department of Genetics, School of Medicine, Yale University,

$\neg \quad$ New Haven, CT, USA. ${ }^{23}$ Department of Computer Science, School of Engineering \& Applied Science, Yale University, New Haven, CT, USA. ${ }^{24}$ MRC Laboratory of Molecular Biology, Cambridge Biomedical Campus, Cambridge, UK. ${ }^{25}$ The Broad Institute of Harvard and MIT, Cambridge, MA, USA. ${ }^{26}$ Facultad de Ciencias, National Autonomous University of Mexico (UNAM), Coyoacán, Mexico.

${ }^{27}$ Priority Organization for Innovation and Excellence, Kumamoto University, Kumamoto, Japan. ${ }^{28}$ Department of Chemical Engineering and Biotechnology, University of Cambridge, Cambridge, UK. ${ }^{29}$ Sao Paulo University (USP), Butanta, Sao Paulo, Brazil. ${ }^{30}$ Laboratory of Biological Engineering, ETH Zurich, Basel, Switzerland. ${ }^{31}$ The Department of Chemistry, University of Basel, Basel, Switzerland. ${ }^{32}$ University of Pennsylvania Perelman School of 
Medicine, Philadelphia, PA, USA. ${ }^{33}$ Corporal Michael J. Crescenz VA Medical Center, Philadelphia, PA, USA. ${ }^{34}$ Department of Biomedical Engineering, Institute for Health Innovation \& Technology, National University of Singapore, Singapore, Singapore. ${ }^{35}$ Department of Surgery, Institute for Health Innovation \& Technology, National University of Singapore, Singapore, Singapore. ${ }^{36}$ Department of Chemical Engineering, Columbia Stem Cell Initiative, Columbia University, New York, NY, USA. ${ }^{37}$ Department of Genetics and Development,
Columbia Stem Cell Initiative, Columbia University, New York, NY, USA. ${ }^{38}$ Department of Bioengineering, Northeastern University, Boston, MA, USA.

${ }^{39}$ Barnett Institute, Northeastern University, Boston, MA, USA. ${ }^{40}$ Single Cell Proteomics Center, Northeastern University, Boston, MA, USA.

${ }^{41}$ Division of Gastroenterology, Hepatology and

Nutrition, Cincinnati Children's Hospital, Cincinnati, OH, USA. ${ }^{42}$ Grousbeck Gene Therapy Center, Mass Eye and Ear, Boston, MA, USA.

${ }^{43}$ Harvard Medical School, Boston, MA, USA.
${ }^{44}$ Center of Excellence in Genomics \& Systems Biology, International Crops Research Institute for the Semi-Arid Tropics, Patancheru, India. ${ }^{45}$ State Agricultural Biotechnology Centre, and Centre for Crop Research Innovation, Murdoch University, Murdoch, Australia. ${ }^{46}$ School of Life Sciences, Tsinghua University, Beijing, China.

Published online: 10 March 2021

https://doi.org/10.1038/s41587-021-00847-1 